\title{
Polarization beam splitting using a birefringent graded photonic crystal
}

\author{
Eric Cassan, ${ }^{1, *}$ Khanh Van Do, ${ }^{1}$ Jean Dellinger, ${ }^{2}$ Xavier Le Roux, ${ }^{1}$ Frédérique de Fornel, ${ }^{2}$ and Benoit Cluzel ${ }^{2}$ \\ ${ }^{1}$ Institut d'Electronique Fondamentale, Université Paris-Sud, CNRS, Orsay 91405, France \\ ${ }^{2}$ Groupe d'Optique de Champ Proche, Laboratoire Interdisciplinaire Carnot de Bourgogne, \\ UMR 6303 CNRS, 9 Avenue A. Savary, Dijon 21078, France \\ *Corresponding author: eric.cassan@u-psud.fr
}

Received September 4, 2012; revised November 14, 2012; accepted December 23, 2012; posted January 7, 2013 (Doc. ID 175232); published February 8, 2013

\begin{abstract}
The use of a birefringent graded photonic crystal (GPhC) is proposed for the realization of an efficient polarization beam splitter. This approach allows decoupling the two functions of efficient light injection for both polarizations and TE/TM beam splitting. A smooth light polarization splitting is naturally achieved due to the different curved trajectories followed within the graded medium by the TE and TM waves. A $160 \mathrm{~nm}$ operating bandwidth with insertion loss around $1 \mathrm{~dB}$ and interpolarization crosstalk below $-15 \mathrm{~dB}$ is predicted by a finite difference time domain simulation. The unusually exploited electromagnetic phenomena are experimentally evidenced by scanning near-field optical measurements performed on samples fabricated using the silicon on insulator photonics technology. These experimental works open perspectives for the use of birefringent GPhCs to manage polarization diversity in silicon photonic circuits. (c) 2013 Optical Society of America

OCIS codes: $\quad 050.5298,160.3918,260.5430,230.1360$.
\end{abstract}

With the on-going continuing development of integrated silicon photonics, the need for managing light polarization has become an important issue. Several TE/TM polarization-independent schemes of photonic circuits have been proposed relying, for example, on the use of square silicon on insulator (SOI) nanowaveguides. From a fabrication point of view, this approach still imposes the control of waveguide and device dimensions with accuracies below a few nanometers and is thus not easily amenable to applications. The main alternative approach to this direct strategy is called polarization diversity. It consists in separating the two light polarizations, managing them separately, and/or converting one polarization into the other one and recombining them after [1]. In this perspective, the key elements to enable polarization diversity for future ultrahigh speed coherent lightwave systems relying on the optical phase control, as in quadrature phase-shift keying receivers, are the on-chip coupling system and the polarization splitting/ rotating elements $[1,2]$.

Due to the strong index contrast between the waveguide core $\left(\mathrm{Si}\right.$ ) and cladding (air or $\mathrm{SiO}_{2}$ ), the TE/TM polarization modal birefringence in nonsquare SOI optical waveguides is naturally large. This property has been exploited for the design of various polarization beam splitters (PBS) based on directional couplers (DCs), whose coupling gaps and lengths were optimized to drop only one polarization on the DC cross port $[3,4]$. One drawback of this approach is related to the tightly required control of interwaveguide gaps, coupling lengths, and waveguide cross-sections to ensure reasonable bandwidth, low interpolarization crosstalk, and low insertion loss [2].

Another approach to design PBS is based on the use of planar photonic crystals (PhCs) that are known to have strongly birefringent properties. Several solutions have been explored in this direction, including the use of the superprism effect [5], polarization-dependent refraction []ㅡ, and bandgap frequency sensitivity to light polarization $[\underline{7}, 8]$. As mentioned in [] $]$, the straightforward implementation of this concept for the design of PBS proved to be ineffective. The reason for this is due to in-plane diffraction frequently occurring due to the grating-like interface between the optical slab waveguide and the two-dimensional (2D) PhC area. In addition to this, optical beams tend to significantly spread into the corrugated medium. The most effective adopted approach to circumvent these drawbacks was to consider $\mathrm{PhC}$ heterostructures with all PhCs operating in a self-collimating regime in addition to the bandgap property for TE/TM splitting $[\underline{7}, \underline{8}]$.

Here we propose another approach to distinguish the two functionalities of light injection and splitting of the two polarizations. The idea is to introduce a graded $\mathrm{PhC}$ (GPhC) in which light is efficiently coupled whatever the polarization is, but allowing a smooth polarization splitting due to the introduced gradual chirp of the crystal structure. To the best of our knowledge, this is the first time that GPhCs, previously introduced in $[9,10]$, are considered for their birefringence properties. This proposal is similar to the one of [11] given for homogenized dielectric metamaterials but is formulated here for GPhCs.

Our design strategy was to identify a possible configuration leading to different light-bending properties for the TE and TM polarizations, respectively. We choose for this a $45^{\circ}$-rotated square lattice $\mathrm{GPhC}$ with a chirped air-hole filling factor. To minimize out-off-plane scattering losses, we choose to operate in band 1 for the two polarizations, but in the close first Brillouin zone area in reciprocal space for TE-like modes and near the bandgap between band 1 and band 2. Considering an SOI wafer with a silicon film thickness of $260 \mathrm{~nm}$, effective indices of 2.95 and 2.267 were considered in TE and TM polarizations. Figures 1 (a) and 1 (b) show, for the considered GPhC configuration, the calculated steady-state fields calculated at $\lambda=1520 \mathrm{~nm}$ using $2 \mathrm{D}$ finite difference time domain (FDTD) simulation for the TE and TM light polarizations, respectively. For both polarizations, light is injected from the slab optical medium at a zero input incident angle. A $2 \mathrm{D}$ chirped hole radius $r / a$ given 
(a)

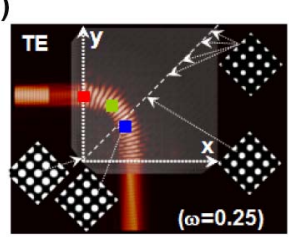

(c)

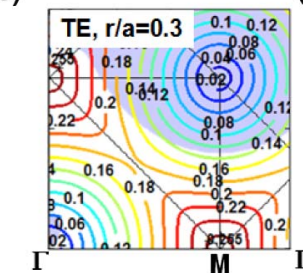

(e)

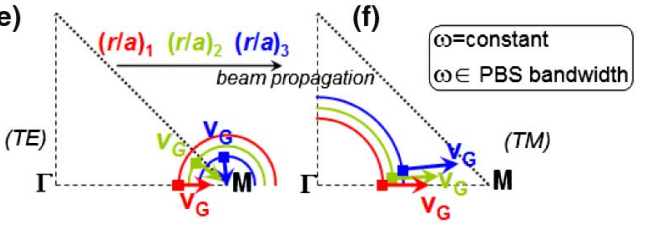

Fig. 1. (Color online) GPhC PBS operating principle. (a) and (b) TE and TM steady-state fields calculated using 2D-FDTD simulation at $\omega=0.25$ for the considered GPhC structure characterized by a $45^{\circ}$-rotated square PhC lattice with $r / a=$ $0.35 \times \exp \left[-\left(x^{2}+y^{2}\right) / 2 R^{2}\right]$. (c) and (d) TE and TM EFS of the related square lattice PhC with $r / a=0.3$. (e) and (f) Schematic picture of the TE and TM EFS at a constant frequency within the PBS bandwidth for $r / a$ values taken along the beam propagation.

by $r / a=0.35 \times \exp \left[-\left(x^{2}+y^{2}\right) / 2 R^{2}\right]$ (but limited to 0.20 ) was considered, with a lattice parameter $a=380 \mathrm{~nm}$ and $R=160 a$. The present useful structure footprint is around $60 \mu \mathrm{m} \times 40 \mu \mathrm{m}$, but simulations showed that it could be reduced at least by a factor of 4 with no degradation of performances. The injection point at $(x=0$, $y=R / 2$ ) was adjusted using ray propagation following the equations of Hamiltonian optics and the local TE photonic band structure. As experimentally evidenced in [12] [and shown here in Fig. 1(a)], a strong $90^{\circ}$-bending effect toward the largest air-hole region is observed for TE waves. At the same time, the TM light polarization fairly propagates straight at the same wavelength [Fig. 1(b)].

The reason for this is related to the fact that the equifrequency surfaces (EFS) of the two polarizations have curvatures of opposite signs with respect to the $\Gamma$ point in reciprocal space in the PBS bandwidth around $\omega=0.25$. As shown in Figs. 1(b) and 1(c), TE-EFS are then centred at the $M$ edge points of the first Brillouin zone and have thus a negative curvature with respect to $\Gamma$, while TM-EFS still have a positive slope with respect to it. The related EFS deformations, wavevector paths, and light group velocities along the propagation of the two polarized beams are schematically represented in Figs. 1(e) and 1(f).

After light injection, the action of the GPhC leads to a smooth polarization beam splitting. With the beam cross-section considered here (waist of $7 a$, i.e., $2.66 \mu \mathrm{m}$, leading to a wavevector spread of $0.032[2 \pi / a]$ ), Figs. 1(a) and 1(b) show that TE and TM optical beams remain almost collimated along the two curved paths. The transmission spectra in the bended and straight channels

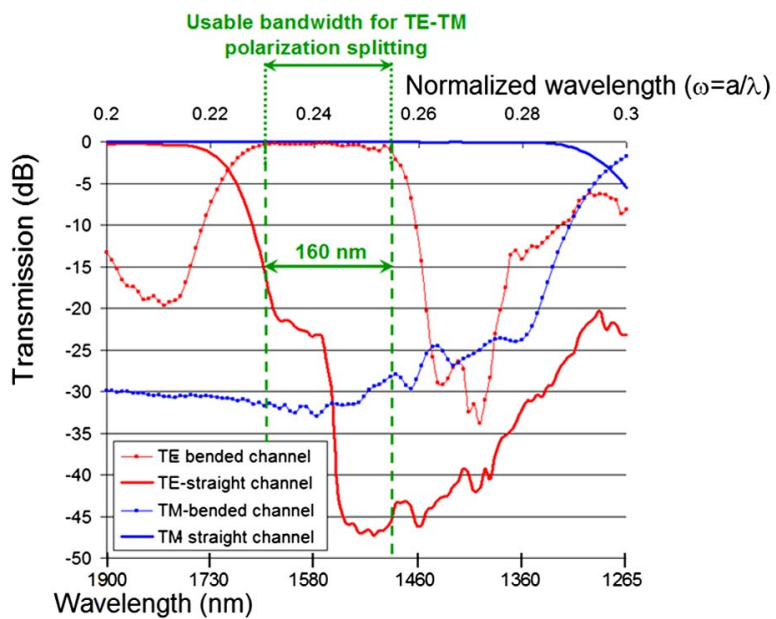

Fig. 2. (Color online) Transmission spectra of the straight and bended channels calculated using FDTD simulation.

have been estimated using FDTD simulation in order to evaluate the polarization crosstalk and insertion losses (see Fig. 2).

In almost the full considered spectrum, TM-waves are collected in the straightforward direction, as shown in Fig. 1(b). At the same time, a high-level transmission window for TE-waves in the bended channel is visible from $\omega \approx 0.23(\lambda \approx 1650 \mathrm{~nm})$ to $0.255(\lambda \approx 1490 \mathrm{~nm})$. In this $160 \mathrm{~nm}$ wavelength range, the TE/TM interpolarization crosstalk in the straight channel is below $-15 \mathrm{~dB}$ and the TM/TE crosstalk in the bended one is below $-27 \mathrm{~dB}$. Considering a still reasonably reduced PBS bandwidth of $70 \mathrm{~nm}(\omega=0.243-0.2545)$, a reduction of the TE/TM crosstalk to $-27 \mathrm{~dB}$ is also obtained. For both channels, the predicted insertion loss level of transmitted waves is below $1 \mathrm{~dB}$ thanks to the use of optimized input/output optical tapers. Although 2D simulation does not account for out-of-plane leakage, the low level of insertion losses was confirmed by far-field experiments performed in TE polarization providing losses below $2 \mathrm{~dB}$ [12]. Additionally, channel backscattering was numerically estimated here to $-22 \mathrm{~dB}$ and $-10 \mathrm{~dB}$ for the TM and TE injected waves, i.e., to lower values than previously reported [7,8].

These birefringent properties were experimentally studied. Fabrication of the GPhC sample contained two stages. First, strip waveguides were defined using a RAITH150 electron beam lithography process using negative resist. The GPhC structure was then insolated by a lithography process with a positive resist, patterns being transferred to a $150 \mathrm{~nm}$ thick top silica-cladding layer using reactive ion etching. This layer served as a mask to etch the silicon film through a $\mathrm{SF}_{6} / \mathrm{O}_{2}$ anisotropic etching process. Figure 3 shows the obtained fabricated structure with a detailed scanning electron microscope (SEM). The views are of the PhC lattice at different locations.

Light paths for TE and TM polarized beams inside were directly visualized by using a scanning near-field optical microscope (SNOM) operating in a collection mode [13]. The experimental SNOM images recorded

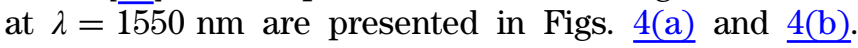
As predicted earlier, the TE-beam is bended inside the GPhC while the TM-beam goes straight. Since the 


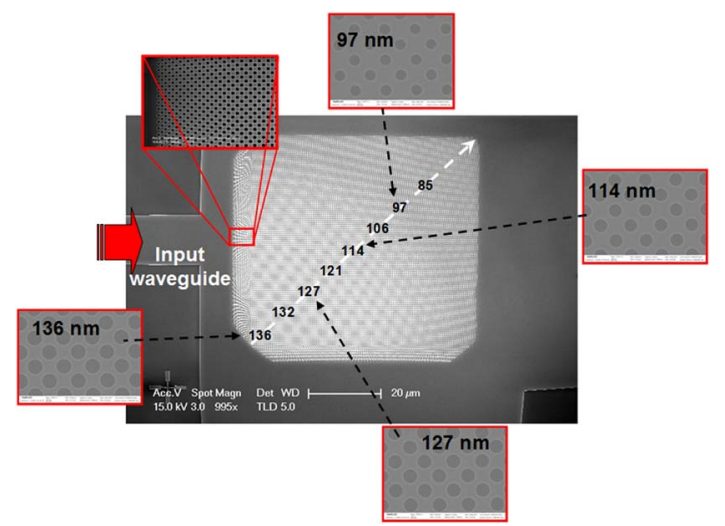

Fig. 3. (Color online) SEM views of the fabricated GPhC structure with detailed views of the lattice at different locations within the graded periodical dielectric medium.

near-field probe is selectively sensitive to the components of the electric field transverse to the probe axis, the TM-beam (major E-field parallel to the probe axis) is measured experimentally with a significantly lower signal-to-noise ratio compared to the TE-beam. This does not prevent the qualitative demonstration of the polarization splitting effect but implies that an attempt to quantitatively compare the data recorded for the TE and TM beams would be hazardous.

At last, the bandwidth of the polarizing beam splitter made of GPhC is quantified by using a hyperspectral SNOM (Hyp-SNOM) [14]. To provide direct evidence of the beam splitting effect over the spectrum, we injected an unpolarized supercontinuum laser source inside the $\mathrm{GPhC}$ and recorded the spectra of the light collected by the probe on each pixel. The SNOM ranging from 1450 to $1600 \mathrm{~nm}$ is available online as a movie and selected snapshots of the hyperspectral measurement at $\lambda=1450,1500,1550$, and $1600 \mathrm{~nm}$, are plotted in Figs. 4(c)-4(f). As seen, the predicted beam splitting effect is evidenced over the full $150 \mathrm{~nm}$ spectral range, while, due to the probe collection efficiency, the TE-beam is more intense than the TM beam. We also note that the TM beam remains almost unchanged over the spectrum while the TE beam bending depends on the wavelength resulting in a variation of its output position. One could benefit from this feature to combine the beam splitting effect with a wavelength division demultiplexing. Otherwise, an inverted tapered waveguide could be used to collect the TE beam and cancel this dispersive effect.

To conclude, the use of graded PhCs for polarization beam splitting is proposed. With respect to previous solutions, the interest of this approach relies on the smooth separation of polarized beams by the gradual medium after efficient injection of light for the two polarizations. A $160 \mathrm{~nm}$ operating bandwidth and a TE/TM crosstalk below $-15 \mathrm{~dB}$ are predicted, with insertion loss around $1 \mathrm{~dB}$. The exploited phenomena are evidenced using

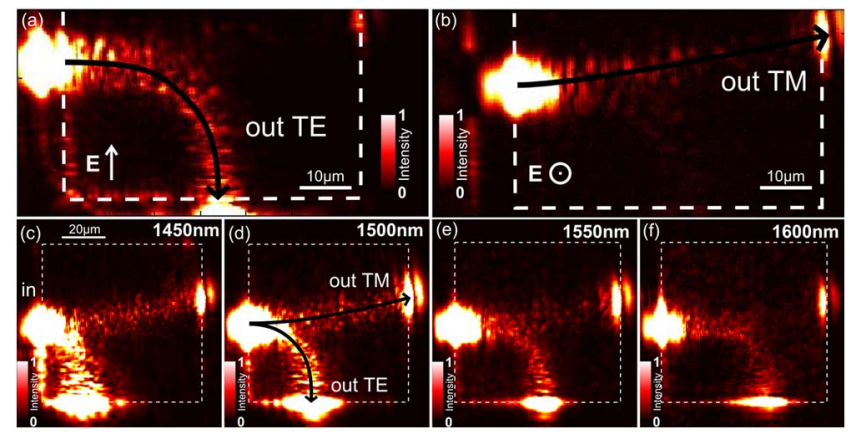

Fig. 4. (Color online) (a) SNOM image obtained at $\lambda=$ $1550 \mathrm{~nm}$ for a TE polarization of the input light. (b) SNOM image obtained at $\lambda=1550 \mathrm{~nm}$ for a TM polarization of the input light. (c)-(f) Hyp-SNOM images obtained at $\lambda=1450,1500,1550$, and $1600 \mathrm{~nm}$ with a nonpolarized laser. The beam splits into TE and TM polarized beams. Over a broadband of $150 \mathrm{~nm}$, the TE-beam is bended while the TM-beam goes straight (Media 1).

SNOM measurements in the $1450-1600 \mathrm{~nm}$ wavelength range. The reported images enable the validation of the predicted phenomena. These works open perspectives for the use of birefringent GPhCs to TE/TM-sensitively curve the flow of light and design optical functionalities for future polarization diversity photonic circuits.

The authors acknowledge the Burgundy Regional Council from France for support (PHOTCOM project).

\section{References}

1. H. Fukuda, K. Yamada, T. Tsuchizawa, T. Watanabe, H. Shinojima, and S.-I. Itabashi, Opt. Express 16, 4872 (2008).

2. T. Barwicz, M. R. Watts, M. A. Popovic, P. T. Rakich, L. Socci, F. X. Kartner, E. P. Ippen, and H. I. Smith, Nat. Photonics 157 (2007).

3. I. Kiyat, A. Aydinlin, and N. Dagli, IEEE Photonics Technol. Lett. 17, 100 (2005).

4. D. Dai and J. E. Bowers, Opt. Express 19, 10940 (2011).

5. L. Wu, M. Mazilu, J.-F. Gallet, T. F. Krauss, A. Jugessur, and R. M. De La Rue, Opt. Lett. 29, 1620 (2004).

6. X. Ao, L. Liu, L. Wosinski, and S. He, Appl. Phys. Lett. 89, 171115 (2006).

7. E. Schonbrun, Q. Wu, W. Park, T. Yamashita, and C. J. Summers, Opt. Lett. 31, 3104 (2006).

8. V. Zabelin, L. A. Dunbar, N. Le Thomas, R. Houdré, M. V. Kotlyar, L. O'Faolain, and T. F. Krauss, Opt. Lett. 32, 530 (2007).

9. P. S. J. Russel and T. A. Birks, J. Lightwave Technol. 17, 1982 (1999).

10. E. Centeno and D. Cassagne, Opt. Lett. 30, 2278 (2005).

11. A. J. Danner, T. Tyc, and U. Leonhardt, Nat. Photonics 5, 357 (2011).

12. K.-V. Do, X. Le Roux, D. Marris-Morini, L. Vivien, and E. Cassan, Opt. Express 20, 4776 (2012).

13. J. Dellinger, D. Bernier, B. Cluzel, X. Le Roux, A. Lupu, F. de Fornel, and E. Cassan, Opt. Lett. 36, 1074 (2011).

14. J. Dellinger, K. V. Do, X. Le Roux, F. de Fornel, E. Cassan, and B. Cluzel, Appl. Phys. Lett. 101, 141108 (2012). 